Supporting Information:

\title{
Anti-Stokes Light Scattering Mediated by Electron Transfer Across a Biased Plasmonic Nanojunction
}

Shuyi Liu ${ }^{1}$, Adnan Hammud ${ }^{2}$, Martin Wolf ${ }^{1}$, Takashi Kumagai ${ }^{1,3 *}$

${ }^{1}$ Department of Physical Chemistry, Fritz-Haber Institute of the Max-Planck Society, Faradayweg 4-6, 14195 Berlin, Germany

${ }^{2}$ Department of Inorganic Chemistry, Fritz-Haber Institute of the Max-Planck Society, Faradayweg 4-6, 14195 Berlin, Germany

${ }^{3}$ Center for Mesoscopic Sciences, Institute for Molecular Science, Okazaki 444-8585, Japan

*Corresponding author: kuma@fhi-berlin.mpg.de; kuma@ims.ac.jp 
1. Incident laser power dependence of anti-Stokes continuum in an electrically-biased STM junction

2. Bias polarity dependence of the anti-Stokes continuum in an electrically-biased STM junction

3. Wavelength dependence of the anti-Stokes continuum in an electrically-biased STM junction

4. Tip dependence of the anti-Stokes continuum in an electrically-biased STM junction

5. Bias voltage dependence of the Stokes continuum in an electrically-biased STM junction

6. Tunneling current dependence of the Stokes continuum in an electrically-biased STM junction 


\section{Incident laser power dependence of anti-Stokes continuum in an electrically-biased STM junction}

As mentioned in the main text, two-photon excitation process to yield the anti-Stokes continuum is excluded by the power dependent measurement. Figure S1a shows the ILS spectra (anti-Stokes regime) obtained for a different irradiation power. During the measurement the tip-surface distance was kept constant and the tip condition did not change. All the spectra show the same shape but different intensity, which linearly depends on the incident power as shown in Fig. S2b.
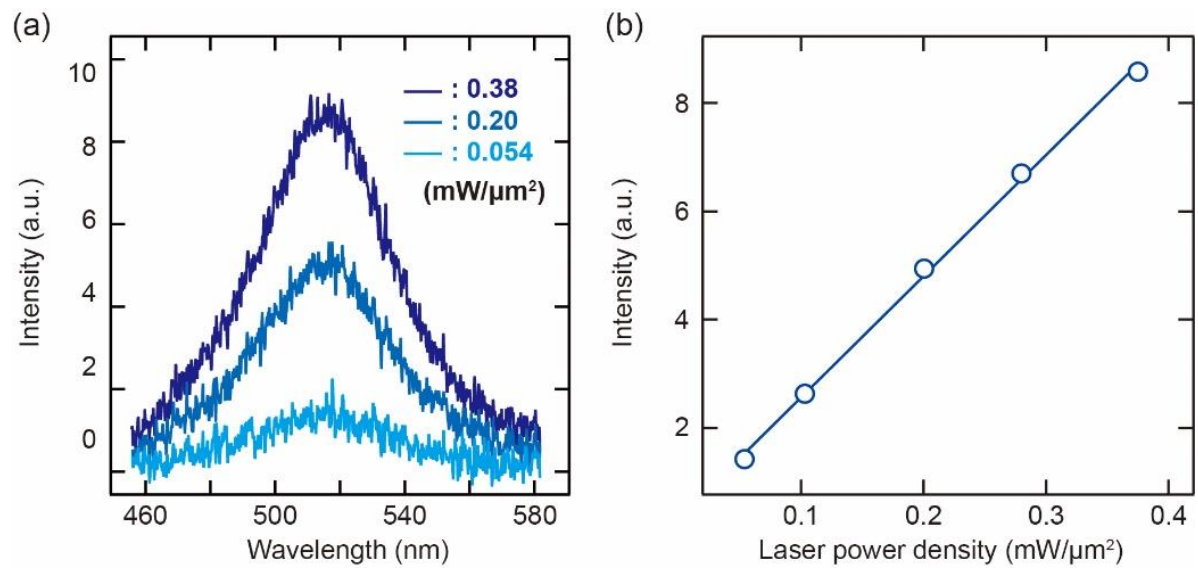

Figure S1. (a) Anti-Stokes regime of the ILS spectra measured for an Ag tip-vacuum-Ag(111) junction at different incident laser powers $\left(\lambda_{\text {ext }}=633 \mathrm{~nm}, j_{\mathrm{t}}=80 \mathrm{nA}, V_{\mathrm{s}}=1 \mathrm{~V}, t_{\mathrm{exp}}=30 \mathrm{~s}, 10 \mathrm{~K}\right)$. (b) Incident laser power dependence of the anti-Stokes continuum intensity. 


\section{Bias polarity dependence of the anti-Stokes continuum in an electrically-biased STM junction}

The anti-Stokes continuum shows no polarity dependence, which is consistent with the proposed ILS mechanism in the main text (Fig. 4). As shown in Fig. S2, the onset coincides with $V_{\text {bias }}$ at a lower voltage $( \pm 0.2 \mathrm{~V})$, whereas the spectral response becomes similar with the one in the STL spectrum at a higher voltage $( \pm 1 \mathrm{~V})$. This is consistent with the $V_{\text {bias-dependence }}$ discussed in the main text.

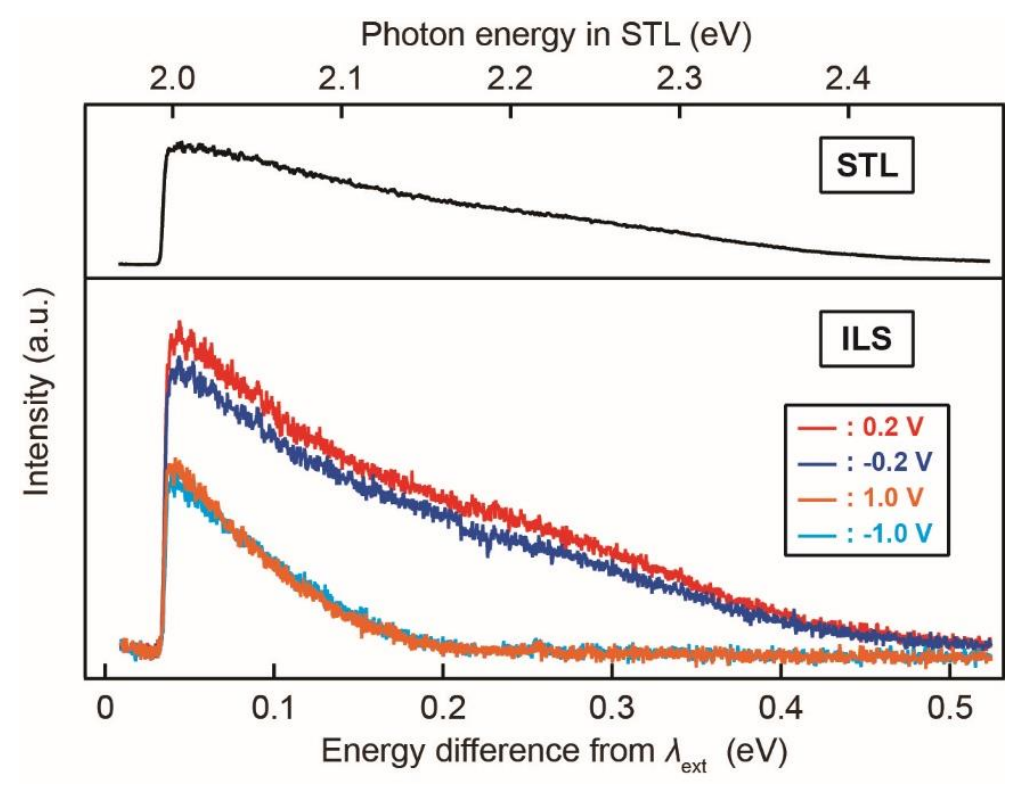

Figure S2. (Top) STL spectrum of an $\mathrm{Ag}$ tip $-\operatorname{Ag}(111)$ junction $\left(V_{\text {bias }}=3 \mathrm{~V}, j_{\mathrm{t}}=6 \mathrm{nA}, t_{\exp }=150\right.$ $\mathrm{s}, 10 \mathrm{~K})$. (Bottom) ILS spectra (anti-Stokes branch) recorded at different $V_{\text {bias }}$ at $j_{\mathrm{t}}=60 \mathrm{nA}$ $\left(\lambda_{\text {ext }}=633 \mathrm{~nm}, P_{\text {ext }}=0.34 \mathrm{~mW} \mu \mathrm{m}^{-2}, t_{\mathrm{exp}}=60 \mathrm{~s}, 10 \mathrm{~K}\right)$. The horizontal axis is the energy difference relative to the excitation photon energy $(1.96 \mathrm{eV})$. 


\section{Wavelength dependence of the anti-Stokes continuum in an electrically-biased STM junction}

As shown in Fig. S3, the anti-Stokes continuum show the same behavior under irradiation at $\lambda_{\text {ext }}=633$ and $532 \mathrm{~nm}$, which is consistent with the proposed ILS mechanism in the main text (Fig. 4). The onset of the continuum is coincident with Vbias at lower voltages $(<0.5 \mathrm{~V})$, whereas the spectral shape becomes similar with the one in the STL spectrum at a higher voltage $(1 \mathrm{~V})$.
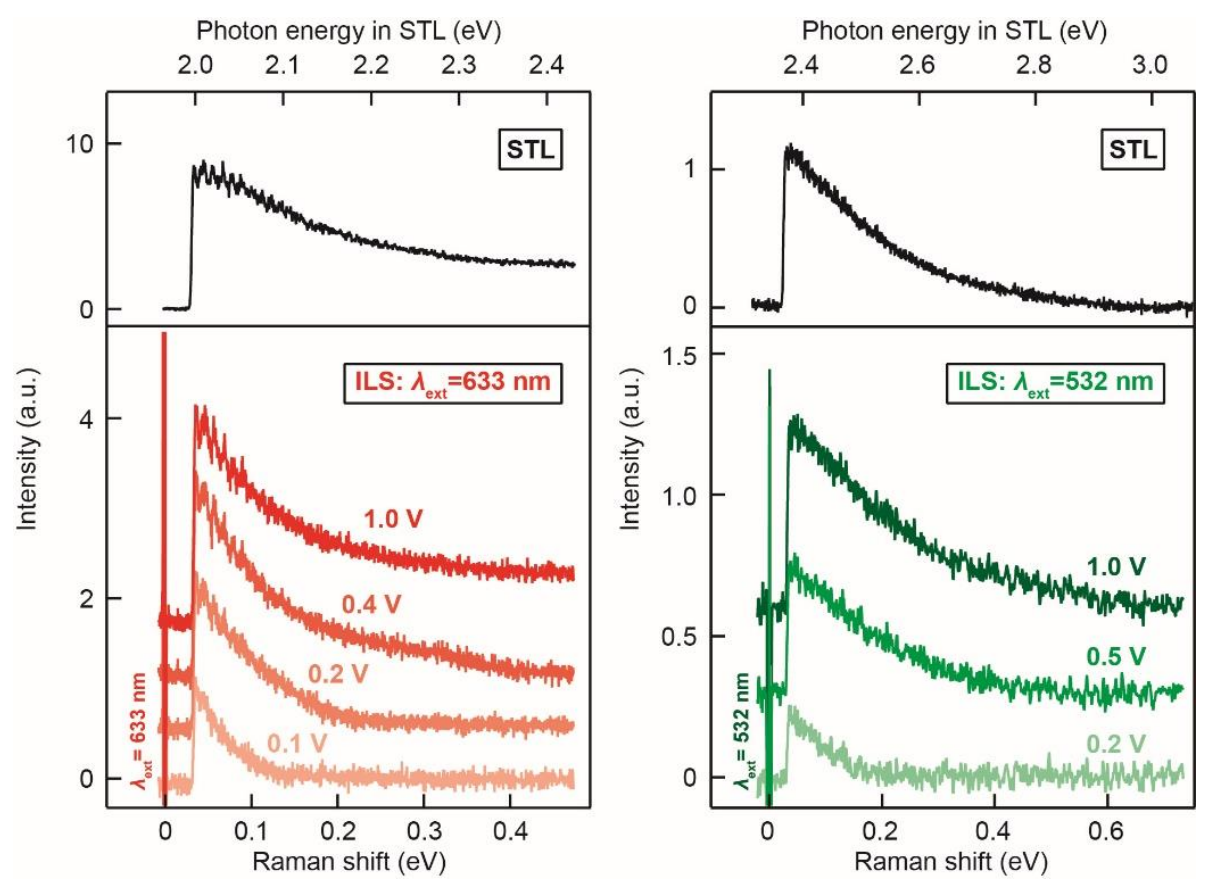

Figure S3. STL (top) and ILS (bottom) spectra measured for the Ag tip-vacuum-Ag(111) junction at a different excitation wavelength. (a) STL: $j_{\mathrm{t}}=8 \mathrm{nA}, V_{\mathrm{s}}=3 \mathrm{~V}, t_{\mathrm{exp}}=90 \mathrm{~s}$. ILS: $\lambda_{\mathrm{ext}}=633$ $\mathrm{nm}, P_{\text {ext }}=0.37 \mathrm{~mW} \mu \mathrm{m}^{-2}, t_{\mathrm{exp}}=90 \mathrm{~s}, j_{\mathrm{t}}=80 \mathrm{nA}, 10 \mathrm{~K}$. (b) STL: $j_{\mathrm{t}}=8 \mathrm{nA}, V_{\mathrm{s}}=3 \mathrm{~V}, t_{\mathrm{exp}}=15 \mathrm{~s}$. ILS

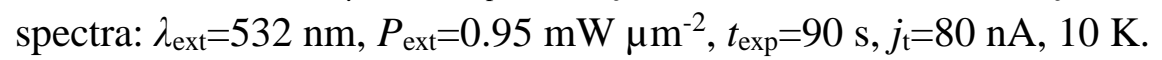




\section{Tip dependence of the anti-Stokes continuum in an electrically-biased STM junction}

As discussed in the main text, the field enhancement acts only on the outgoing field in STL and thus the intensity $\left(I_{\mathrm{STL}}\right)$ follows $I_{\mathrm{STL}(\lambda)} \propto g(\lambda)^{2}$, whereas in ERS it follows $I_{\mathrm{ERS}\left(\lambda_{\text {ext }}\right)} \propto$ $g\left(\lambda_{\text {ext }}\right)^{2} \cdot g(\lambda)^{2}$ because the field enhancement also occurs for the incoming field $\left(\lambda_{\text {ext }}\right)$. According to these relations, we can derive the following relation:

$$
\frac{I_{\mathrm{STL}, \mathrm{tip} \# 4\left(\lambda_{\text {ext }}\right)}}{I_{\mathrm{STL}, \mathrm{tip} \# 5\left(\lambda_{\text {ext }}\right)}} \cdot \frac{I_{\mathrm{STL}, \mathrm{tip} \# 4(\lambda)}}{I_{\mathrm{STL}, \mathrm{tip} \# 5(\lambda)}}=\frac{I_{\mathrm{ILS}, \mathrm{tip} \# 4(\lambda)}}{I_{\mathrm{ILS}, \mathrm{tip} \# 5(\lambda)}}
$$

If the LSPR in the junction is resonant with the $\lambda_{\text {ext }}$, ERS is dominated, which is the case for tip \#4 and tip \#5. In Fig. S4, $R_{1} \equiv \frac{I_{\mathrm{STL}, \mathrm{tip} \# 4\left(\lambda_{\mathrm{ext}}=633 \mathrm{~nm}\right)}}{I_{\mathrm{STL}, \mathrm{tip} \# 5\left(\lambda_{\mathrm{ext}}=633 \mathrm{~nm}\right)}}=2.4( \pm 0.1), R_{2} \equiv \frac{I_{\mathrm{STL}, \mathrm{tip} \# 4(\lambda)}}{I_{\mathrm{STL}, \mathrm{tip} \# 5(\lambda)}}=$ $3.3( \pm 0.1)$ and $R_{3} \equiv \frac{I_{\mathrm{ILS}, \mathrm{tip} \# 4(\lambda)}}{I_{\mathrm{ILS}, \operatorname{tip} \# 5(\lambda)}}=7.2( \pm 0.1)$ at $\lambda=610 \mathrm{~nm}$, hence $R_{1} \cdot R_{2}=7.9 \approx R_{3}$, thus satisfying the equation (1) that supports the predominant ERS process. However, at $\lambda=$

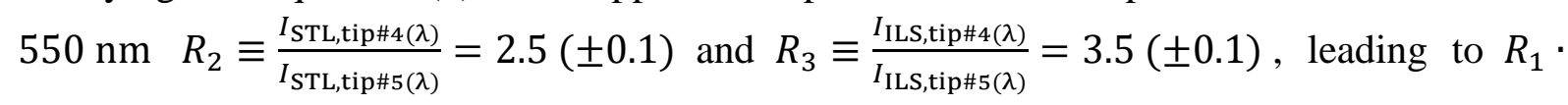
$R_{2}=6>R_{3}$. This deviation may imply that more electrons are transferred through the PL process because of the smaller cross section of ERS resulting from a lower energy of the final state (it is lower by $0.22 \mathrm{eV}$ at $\lambda=550 \mathrm{~nm}$ than that at $\lambda=610 \mathrm{~nm}$ ). According to the model in Fig. 4, the wave function of $|f\rangle$ would less extended into the vacuum barrier if its energy is further below the vacuum level, which results in a smaller overlap of wave functions between $|i\rangle$ and $|\mathrm{f}\rangle$, thus, reducing the Raman cross section. A measurement artifact, such as, plasmon response shift of the junction due to different STM set points, would also respond to the deviation. PL is dominated for tip \#7, tip \#8, tip \#9. The intensity of STL around $\lambda_{\text {ext }}=633 \mathrm{~nm}$ is $I_{\mathrm{STL}, \mathrm{tip \# 7}}>I_{\mathrm{STL}, \mathrm{tip \# 8}}>I_{\mathrm{STL}, \mathrm{tip \# 9}}$, whereas the intensity of the ILS spectra around $\lambda_{\text {ext }}$ is $I_{\text {ILS,tip\#9 }}>I_{\text {ILS,tip\#7 }}>I_{\text {ILS,tip\#8. The spectral shape in STL for tip \#7 and tip \#9 is similar as }}$ their corresponding ILS spectra. However, it deviates obviously for tip \#8. This weak correlation between the STL and ILS spectra is because the hot electrons can be excited by the LSPR at the tip base in addition to the tip apex as discussed in the main text. In tip \#6, both ERS and PL may be contribute equally because its plasmonic response around $\lambda_{\text {ext }}$ is in-between the two cases where either ERS or PL dominates the process. 

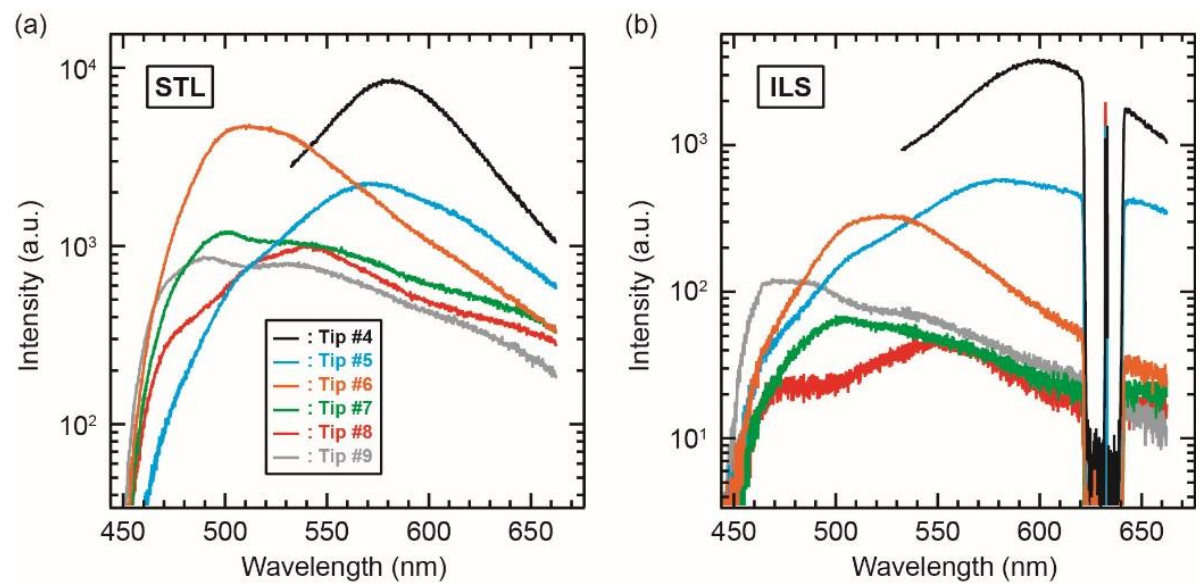

Figure S4. (a) STL measured with various tips ( $\left.V_{\mathrm{bias}}=3 \mathrm{~V}, j_{\mathrm{t}}=3 \mathrm{nA}, 10 \mathrm{~K}, t_{\exp }=30 \mathrm{~s}\right)$ (b) ILS spectra of the anti-Stokes regime measured with the corresponding tips in (a) $\left(\lambda_{\text {ext }}=633 \mathrm{~nm}\right.$,

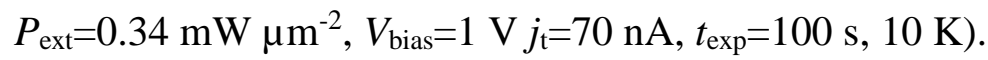




\section{Bias voltage dependence of the Stokes continuum in an electrically-biased STM junction}

It was found that the Stokes continuum is also affected by the applied $V_{\text {bias }}$ to the junction. As shown in Fig. S5, the onset of the continuum is associated with the $V_{\text {bias, }}$ which almost coincides with the applied bias $V_{\text {bias }}=0.2 \mathrm{~V}$, but it is obvious smaller than that for $V_{\text {bias }}=0.4 \mathrm{~V}$. This deviation is consistently observed under different tip conditions. This may be attributed to momentum conservation during the ERS process. There is a strict requirement of momentum conservation for the electron transition in a crystal, but it is relaxed for a confined light via the uncertainty principle: $\Delta k \sim(\pi / \Delta x)$, where $\Delta k$ is a momentum difference between the initial and final states, $\Delta x$ is the size of the confined light. This allows energy range of $\sim 0.4 \mathrm{eV}$ for $\Delta x=1$ $\mathrm{nm}$. A lager energy difference in the Stokes regime corresponds to the electrons scattered into higher energy levels above the Fermi level, namely, a lager momentum change, but it is unlikely.

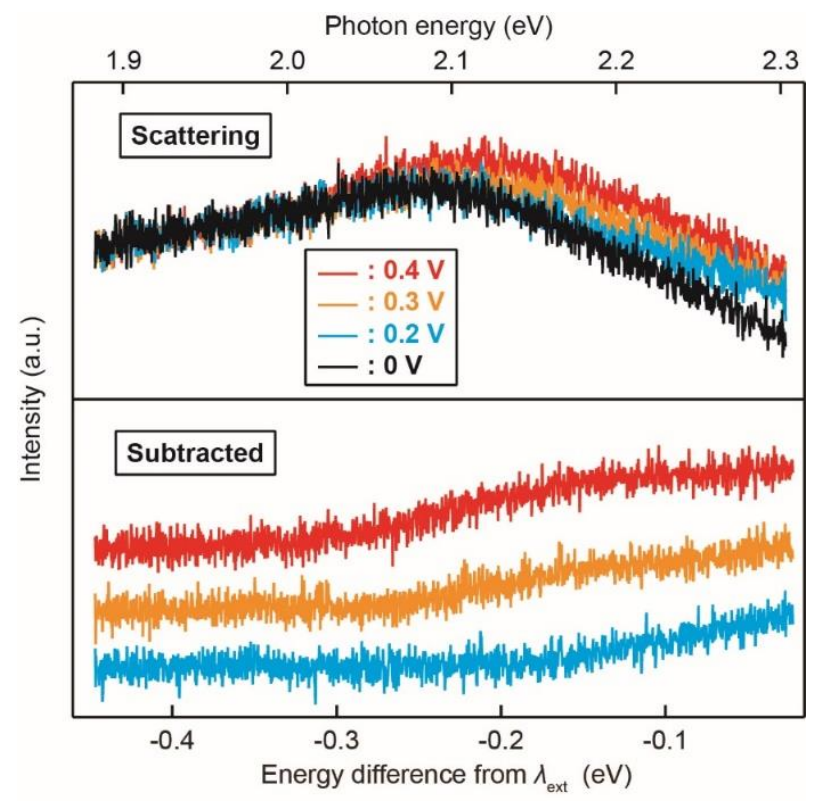

Figure S5. (a) Stokes regime of the ILS spectra from an electrically-biased Ag tip-vacuum$\operatorname{Ag}(111)$ surface junction at different $V_{\text {bias }}$ (indicated in the figure) with the tip height fixed

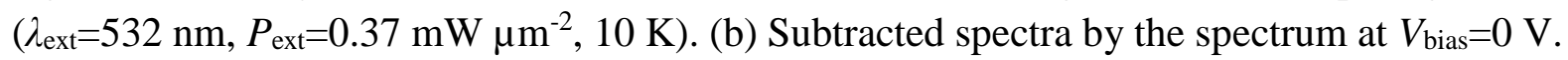




\section{Tunneling current dependence of the Stokes continuum in an electrically-biased STM junction}

Under some tip conditions, the intensity of both Stokes and anti-Stokes branches is saturated simultaneously at high tunneling currents. This saturation is attributable to the quenching of the gap-mode plasmon through quantum mechanical effects.
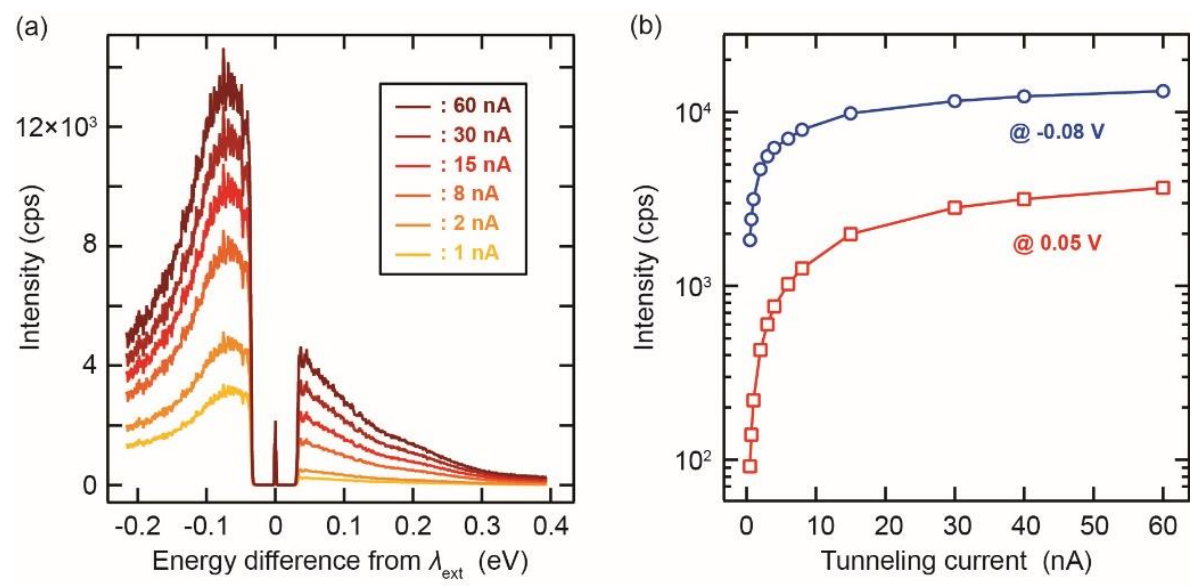

Figure S6. (a) Tunneling current dependence of the ILS spectra measured for an Ag tip$\operatorname{Ag}(111)$ junction $\left(\lambda_{\text {ext }}=532 \mathrm{~nm}, P_{\text {ext }}=0.25 \mathrm{~mW} \mu \mathrm{m}^{-2}, V_{\text {bias }}=0.5 \mathrm{~V}, 10 \mathrm{~K}\right)$. (b) Intensity of the Stokes and anti-Stokes continuum plotted as function of tunneling current. 